\title{
Invited Commentary: Exploring the Promises, Intricacies, and Challenges to Positive Youth Development
}

\author{
Margaret Beale Spencer - Tirzah R. Spencer
}

Received: 18 February 2014/ Accepted: 20 March 2014/Published online: 12 April 2014

(C) Springer Science+Business Media New York 2014

\begin{abstract}
We highlight the need for and critical importance of the program of research reported in this Special Issue. We emphasize that a focus on positive youth development is sorely overdue. The impressive project covering one decade, 42 states and 7,000 participants demonstrates critical contributors to positive youth development. The two identified themes emerging from the set of papers are that contexts and the nature of assets matter for youth. Collectively, the papers explored a variety of positive youth development relevant questions and utilized combinations of annual assessments and data sources from an extraordinary data base. The core methodological and conceptual flaw across papers was the under-representation of minorities in the data set, which limited the generalizability of findings. While the major shortcoming was acknowledged at the onset and recognized as a failing in each paper, nevertheless, merely conceding the flaw was seen as insufficient given the policy, practice, and research implications. The inadequate sampling and followup of youth from families which continue to face persistent social inequality and having the most to gain from a positive youth development conceptual strategy and was a major shortcoming; it prevented within group analyses. The coauthors note that although research decisions made limited the generalizability of the positive youth development research strategy for non-white American youth, the intended goals for inclusiveness are evident and, accordingly, suggest a level of hopefulness.
\end{abstract}

\footnotetext{
M. B. Spencer $(\square)$

University of Chicago, Chicago, IL, USA

e-mail: mbspencer@uchicago.edu

T. R. Spencer

Nemours Health and Prevention Services, Nemours/Alfred I.

duPont Hospital for Children, Newark, DE, USA

e-mail: tirzah.spencer@nemours.org
}

Keywords Context $\cdot$ Assets $\cdot$ Minorities $\cdot$ Positive youth development

\section{Introduction}

Our commentary is divided into three parts. First, we discuss two overlapping themes evident in the research presented in the set of articles of this timely and much needed Special Issue. Second, we present a section which annotates and describes this ambitious set of articles. Third, we highlight methodological and conceptual challenges encountered in the implementation of the 4-H Study of Positive Youth Development (PYD). Across the three parts of our commentary, we both explain that the project is impressive but that it has limitations that have implications for the generalizability of its findings.

\section{Themes in the Special Issue}

Two highly consequential themes emerge from the contributions to this Special Issue, and characterize its conceptual framing. These issues have salience for all teenagers: contexts and assets. Described first in the introductory article by Bowers, Geldhof et al., the dual emphasis of the eight subsequent relational developmental systems-based articles of this special issue each asserts that youth do not develop in a vacuum. Consideration of the contexts they encountered is critical, as adolescents navigate a variety of social spaces and physical places while taking on the myriad developmental requirements mandatory for healthy transition into productive adulthood roles.

As the classic ideas by Havighurst (1953) suggest, individuals' successful coping with meeting developmental 
tasks leads to successful outcomes and well-being. For adolescence, this is positive youth development. Of course, given psycho-historically relevant societal changes, the specifics of each task are different in the twenty-first century from the categorical exemplars described in publications by Havighurst over 60 years ago. Nonetheless, successful coping with tasks is necessary for development, as well as acknowledging the character of activities and myriad assets needed for positive youth development and for the transition into successful adulthood. Both productive coping with tasks and having accessible assets remain salient and relevant for achieving productive outcomes.

Paramount to acknowledge, as well, and as variously described in the studies of this special issue is that youth activities take place in a myriad of settings. The variety of contextual differences described in this special issue include those encountered when early adolescents transition from middle school to secondary school settings (e.g., see Chase et al., in this issue) as well as those reported for youth engaged in out of school-time activities (e.g., see Agans et al., in this issue).

The second of the dual themes emphasized across these reports from 4-H Study of Positive Youth Development (PYD) is the simple truth that assets matter and contribute to positive outcomes and the thriving process. Neither insight (i.e., context and assets) is new, but their "re-visitation" in such a large study, which contains participants from multiple ethnicities, is refreshing. In fact, the 4-H Study researchers' critical transition from a non-deficit perspective, particularly for youth of color, has an important history. To name two exemplars, their consideration was introduced both in the SRCD-supported 1985 edited volume, Beginnings: Social and Affective Development of Black Children (Spencer et al. 1985) and then was reiterated 5 years later in 1990 as a collaborative journal effort in the Child Development Special Issue, Minority Children (Spencer and McLoyd 1990). For the current 4-H Study work, both themes' joint presence in the eight-wave longitudinal effort is expansive and nonpathology in orientation and, accordingly, deserves significant acknowledgement. The application of the relational developmental systems model across studies should be recognized as both nuanced and a forward-directed theoretical formulation.

However, there remain significant conceptual and methodological gaps requiring successful navigation and reflection for maximizing authentic application and use by practitioners, policy makers, programmers, researchers, and socializing agents situated in homes, schools, and communities (i.e., all systems purporting to provide services, opportunities, and support). The Robert Frost poem, which appears at the end of our commentary, "Stopping By Woods on a Snowy Evening," communicates our thoughts about our current scientific and professional circumstance. That is, undoubtedly, we recognize our current situation and dilemma as developmental and behavioral scientists and the destination expected for maximizing youth well-being. Without question, remaining steps include multiple procedural and theoretical spaces to traverse for identifying and asserting the contributors to positive youth development outcomes desired for all youth irrespective of race, ethnicity, immigration, and socio-economic status. However, this goal requires not only comparisons between groups but, also, intra-group analyses.

Enacting a focus on this goal will provide opportunities to ascertain the impact of specific situational spaces as well as the efficacy of particular assets that are intended to be supportive for those for whom they were actually designed to support (e.g., see McGee and Spencer 2012; Spencer 2011). Impressively, the several collaborations represented by the eight research articles utilizing one or more of the eight waves of the same data base represent, as a collective endeavor, authentic progress and provide important lessons. Varied ecological assets have been identified and included in the 4-H Study research. Analysis of the findings offers particular insights about development and, as well, cautions about generalizability long known but infrequently integrated and represented in our conceptual strategies and methodologies. A synopsis of each contribution follows.

\section{A Synopsis of the Contributions of this Special Issue}

The first article by Bowers, Geldhof et al., "Thriving Across The Adolescent Years: A View Of The Issues," provides an effective introduction and overview of the 4-H Study of PYD, an important study of over 7,000 youth surveyed across eight waves with over half of their parents involved, as well. The sample consists of female and male adolescents and includes minority representation, but too few for within group analyses. The authors acknowledge the lack of adequate diversity as a weakness which limits generalizability. The subsequent articles provide multiple illustrations of unique approaches for understanding PYD and the conditions which challenge its full expression.

The article by Callina et al., "Hope in Context: Developmental Profiles of Trust, Hopeful Future Expectations, and Civic Engagement across Adolescence," points to a past lack of focus on parent-child relationships in the development of adolescents' hope. The innovative approach in this study involved seeking to identify developmental profiles of youth "with respect to hopeful future expectations (HFE) and to parental trust across adolescence" (i.e., across Grades 7-10). Among the quite fascinating findings and, contrary to their hypotheses, the profile reflecting the greatest discrepancy in HFE and trust across early to middle adolescence (i.e., Moderate Hope/U-shaped Trust) was associated with the highest mean Contribution scores. 
America is not a post-racial society, although it is comforting for some to infer that the election of the nation's first African American President suggests otherwise. The unavoidable awareness of youth of different "everyday treatment" experienced as a function of race/ ethnicity and gender status differences would suggest potentially varying profiles of HFE by race and such a possibility deserves attention. Race/ethnicity impacts the degree of individual-context fit and of youth perceptions of trustworthiness of settings and individuals. Accordingly, discerning the specific assets (i.e., both as contextual resources and as human characteristics) needed for moderating the adaptation requirements for PYD deserves attention. The study's inability to test whether HFE and trust produce similar profiles for adolescents of color is an unfortunate missed opportunity, which potentially thwarts efforts designed to specify fully positive youth outcomes.

The contribution of Chase et al., "Academic Achievement in the High School Years: The Changing Role of School Engagement," introduces analyses that focus on the construct of school engagement (i.e., the degree to which students are involved in and committed to the academic and social activities in school). The dilemma for education represented by achievement gap findings underscores the importance of the study. The authors, approaching the complex situation from a human development (as compared to an education) perspective, consider the multiple and relevant domains of development, utilize a novel tripartite model emphasizing behavioral, emotional, and cognitive components, and investigate how engagement in the school context relates to academic achievement throughout high school. Given the persistent racial achievement gap findings, the article provides important information. In fact, in contrast to previous efforts, the co-authors wished to explore the reciprocal relationship among factors affecting engagement. Specifically, they sought to determine the magnitude and direction of the relations among students' self-reported GPA and the tripartite components of behavioral, emotional, and cognitive school engagement across the high school years. Findings included the observation that behavioral school engagement was the strongest indicator, suggesting support for the thesis that study skills and effort may contribute to academic success.

Among other study outcomes, cognitive and emotional factors, although certainly influentially linked to behavioral ones, were found to not account for academic success. Feeling good about school or thinking positively about it, does not necessarily translate into the positive behavioral characteristics (e.g., study skills) that are the strongest predictors of academic success. The authors note that this finding may be linked to the relative consistency of academic performance during the high school years. From our perspective, given the biases of the sample, we agree with the authors' caution that the mainly European American sample introduces conceptual challenges. Youth of color too frequently have known about or themselves had personal experiences which are interpreted as evidence that effort does not matter. That is, performing well, subscribing to study skill investment, and demonstrating perseverance of effort, in and of itself, may not be adequate for obtaining the desired academic outcomes. The varied meaning-making processes suggested are legitimate differences which should be included in the identified challenges and assets considered. Thus, although not testable given the noted limitation in the sample, for minority students, evidence countering the authors' reported findings would involve findings that behavioral school engagement indicators may be different or function differently for particular minorities than they do for European American youth who, too frequently, are made to represent the "standard" against which all others are compared.

The article by Bowers et al., "Important Non-Parental Adults And Positive Youth Development Across Mid- To Late-Adolescence: The Moderating Effect Of Parenting Profiles," examines individual thriving (the Five Cs of PYD) across mid- to late-adolescence (i.e., across four waves), and explores how PYD is moderated by youth relationships with parents (as indexed by school involvement, warmth, and monitoring) and with important nonparental adults. The authors identified profiles of perceived parenting and then assessed how the profiles related to the Five Cs and to important non-parental adults. The sample reported included ethnic-diversity challenges which are salient, given the assumptions about minority parenting practices that too often ignore experiences associated with structurally determined, generally unacknowledged, and socially imposed low resource conditions. Such challenges are uniquely evident at all levels of social, psychological, and physical ecologies and are salient for parenting practices. Notwithstanding the conceptual challenges noted, the overall questions posed are important. The results suggest that the majority of youth perceived their parents to exhibit a relatively positive parenting profile. At each wave many youth were members of an Integrative profile; in fact, most youth were actually members of the Authoritative or Highly Involved parenting profiles. As suggested, given the context-independent labeling pattern associated with African American parenting adults in the literature (i.e., as generally authoritarian), the study's shortcoming is its missed opportunity to obtain data important for understanding minority family parenting and positive youth experiences.

The contribution by Agans et al., "Activity Involvement as an Ecological Asset: Profiles of Participation and Youth Outcomes," addressed another important set of questions. The authors explored patterns of adolescent activity involvement and the developmental outcomes associated 
with long-term patterns of participation. The questions were thoughtful and included "whether participation in a wide variety of activities is indeed beneficial for youth development, does such breadth of participation remain adaptive if it is sustained across the high school years, or is it more beneficial for adolescents to begin to reduce the number of activities in which they participate? Furthermore, to what extent is a young person's pattern of participation predictive of developmental outcomes at the end of high school?" The authors found that changes in adolescents' breadth of participation were associated with increased substance use, depression, and other risk behaviors, as well as with lower levels of Contribution. In addition, consistent membership in the high-participation group was associated with higher levels of PYD and Contribution, as well as with lower levels of substance use, in contrast to individuals with consistent membership in the lower-participation group. Generally, there was a lower level of risky behaviors among individuals in the higherparticipation group. Youth from lower-SES families were more likely to move between the high and lower participation groups.

More than likely there were unique reasons for the mobility by social class, which might suggest that the supports and incentives required would also need to be different. The findings by social class would have been more salient if also available by race/ethnicity, given the overall social class findings. On the one hand, and as stated, there is an assumption that middle-income Whites represent the standard and that all minorities experience and interpret contexts similarly, independent of social class status (i.e., an inference that "whiteness" dominates and supersedes other factors). Given the sheer number of study participants, the opportunity to examine the race/ethnicity by social class groupings would have been highly salient for programming and policy implications. That is, if participation opportunities matter across the high school years, consequentially, are particular minority groups, differentiated by socioeconomic class, also in need of greater support for activity participation versus an assumption that all youngsters require and have access to the same level of support? One would infer that from a program and management perspective, a strategy of implementing support-sensitive policy responses for planning purposes represents an economically sound approach; potentially, it would also lessen stereotyping for those, in fact, not needing additional support, although representing a particular racial group membership. In sum, the differentiated orientation we suggest involves supports, overall, which would diminish potential stereotyping.

The authors of the next article, Geldhof et al., "Longitudinal Analysis of a Very Short Measure of Positive Youth Development," researched the bi-factor structure of PYD, as indexed by the Five Cs, across all eight waves of the 4-H study. The authors used the very short 17-item form of the PYD measure (the PYD-VSF), which, due to its brevity, allowed a test of the structure of the bifactor model across all waves of the 4-H Study. Related to this work was their desire to ascertain whether this form might be useful for youth program evaluation. Of course, having an inadequate sample of minorities suggests an inability to infer the form's adequacy and potential appropriate use with groups distinct from European Americans. That critique notwithstanding, the effort is nonetheless important. Their bifactor models suggest strong correlations among several of the five Cs. Among other findings, Competence and Confidence were highly correlated, suggesting that participants who rate themselves as being competent would also rate themselves as having high levels of self-confidence.

Of course for a diverse sample, it would be critical to explore, for example, if the feedback to high Confidence males of color is perceived in the same way; thus, might the construct and experience be different? Character and Caring were also correlated with each other while displaying consistently low correlations with Competence and Confidence. Connection was consistently and strongly correlated with Competence, and Confidence appeared consistently and weakly correlated with Caring. In addition, Connection was moderately to highly correlated with Character early in adolescence but decreased during later adolescence.

The authors conclude that the PYD-VSF can be an efficient and useful measure of youth thriving and for assessing links to positive and difficult features of development across adolescence. They note that the correlations among the PYD score and its indices are not uniformly positive or negative and the nature of these connections changes over the course of adolescence. They suggest that PYD may be a valuable tool for program evaluation, particularly if these observations regarding correlation/changes are incorporated into the analyses. We would add, given the sampling concerns we have stated, that it would be important that a follow-up study, with a larger and representative sample of youth, be conducted for examining whether the outcomes indicated hold with a more diverse sample.

Hershberg et al., the research team for "Illuminating Trajectories of Adolescent Thriving and Contribution through the Words of Youth: Qualitative Findings from the 4-H Study of Positive Youth Development," explored the 4-H Study from a qualitative perspective. Their strategy was to examine the reported words of youth who participated in the 4-H Study in Grades 6, 9, and 12. The authors analyzed participants' responses to two open-ended questions about the most meaningful aspects of their daily lives and their visions of their idealized selves. It is timely to 
recall that longstanding questions remain about the "referent others" that contribute to the delineation of the self for minority youth. Thus, this important study represents another excellent opportunity for understanding diversity, given its potential for reporting diverse youths' unique perceptions and meaning making processes of salient experiences (over time) and the implications of those daily experiences and visions for their multiple selves.

The authors themselves acknowledge the challenge that the participants might define certain PYD concepts differently than views of scholars and practitioners (i.e., thriving may be seen as synonymous with making money for some adolescents). Differences were observed between males and females, with the former group placing greater emphasis on self-improvement, and the latter group more interested in improving the circumstances of peers and communities-both sets of findings having implications for program design and implementation. As suggested previously, given the greater familial interdependent functioning for some cultural minorities, minority status membership would introduce the possibility of even greater variation in the defining and reporting of everyday experience.

In the next article, "Profiles of Problematic Behaviors across Adolescence: Covariations with Indicators of Positive Youth Development," Arbeit et al. wished to examine the relationship between problem behaviors (i.e., depressive symptoms, bullying, delinquency, substance abuse, disordered eating behavior, and problematic sexual behavior) and positive development (the five Cs). Several research questions were pursued: (1) Can profiles of problematic behaviors be identified in Waves 2 through 8 (Grades 6 through 12) of the 4-H Study of PYD?, (2) If yes, then what is the relationship between various profiles of risk behaviors and the five Cs of PYD?, and (3) Are there patterns of gender difference relating to profile membership? Among other outcomes, the authors found higher levels of Confidence and Competence associated with the Low Risk profile and with the Alcohol and Aggression profile. Low levels of Confidence and Competence were consistently found in the Mental Health Struggles and the Mental Health and Other Risks profiles, and low levels of Confidence were found in the High Drive for Thinness profile. The authors suggested that this set of findings reflects a possible association between high levels of Confidence and Competence and externalizing behaviors.

In part, the authors interpreted these findings in the context of research on the role of sports in youth development. Sports participation has been linked to the Five Cs of PYD, and sports participation has been shown to be correlated with alcohol consumption. They contrasted this assessment with the profiles of youth with low Confidence, which is characteristically associated with depressive symptoms and other internalizing symptoms. Males were more likely to fall into the Alcohol and Aggression Group as well as the High Risk group. Females were more likely to be part of the Mental Health Struggles, Mental Health and Other Risks, and High Drive for Thinness groups. The authors note that this set of findings fits into existing literature that indicates males are more likely be delinquent and substance users whereas females are more likely to show signs of depression and eating disorders. The authors noted that participants in the Low Risk group were more likely to stay in the study, whereas those in groups with the highest levels of problematic behaviors were more likely to leave.

From our point of view, these relationships may be more complex among ethnically diverse youth. For example, among Black girls, Black cultural identity is significantly associated with girls' reports of fewer depressive symptoms and not associated with BMI or with overweight concerns. In addition, for minority youth, the use of sports might be interpreted as providing potential access to unusual levels of opportunity. In short, the relationship between PYD and behavioral health profiles may be more nuanced if one considers race and race-class variations.

The next article, by Hillard et al., "The Deficit Model: Bullying and Trajectories of Character Virtues in Adolescence," examined bullying and character in adolescence by studying the development of performance, civic, and moral character in relation to youth self-reported bullying status. The authors explored trajectories and possible interrelationships among moral character, civic character, and performance character trajectories. Then they examined whether the status of bullying/being bullied was differentially associated with the trajectories for the three character types. They hypothesized that bullying would be negatively associated with moral and civic character trajectories, but would be related to performance character trajectories. In addition, they hypothesized that youth not involved in bullying would exceed bullies in civic and moral character domains. No predictions were made regarding bully-victims. Nevertheless, the study is innovative.

\section{Methodological and Conceptual Challenges}

As noted, the studies reported in this special issue asked unusually sensitive and important questions, and the findings are interesting, some novel and quite unexpected, and all suggest critical insights for adults as service providers, teachers, and parental socialization agents. The underrepresentation problem, appropriately, has been acknowledged in the articles. Nonetheless, as we alluded to previously, the twenty-first century national standpoint concerning race attempts to view the nation as post-racial, thus, feigning the view that identifiability by race no longer 
matters. However, the unique contexts experienced by those under-represented in the large scale study should give rise for concern about generalizability. Pointing to this dilemma allows us to highlight the continuing problem of adaptations required by some youth for solving the same developmental tasks or for navigating the same spaces and places. As one candid example, every parent of an adolescent preparing for the ritual of high school-provided Driver Education programming in pursuit of youth obtaining licensure to drive, the implications of "driving while Black or Brown" suggests needed cautions. Our point is that the pursuit and correlates of PYD for adolescents of color require special coping and adaptations with adults, and particularly non-family "others" more generally. Thus, the occasion for a group of studies representing a national data set of over 7,000 youngsters was a bold plan. Its potential for assisting in unraveling the similar and different experiences required for positive youth development was no small goal and deserves special recognition and accolades.

However, as we have suggested, the individual-context "fit" (or not) issue matters deeply and, most importantly, the fact of its salience has a long history but was not enough to ignite concern very early in this ambitious, decade-long, eight waves of data collection in this innovative undertaking on positive youth development. The good news is that the study was conceptualized and implemented, and its findings provide significant scholarship on PYD for European American youth. The disappointing news, of course, is its missed opportunity, on methodological grounds, to obtain insights about and address the PYD needs of a large American minority youth constituency. The situation represents a quite stable conceptual dilemma and shortcoming of extant youth development research.

For a start, ecological perspectives were evident during the latter decades of the first half of the twentieth century and interpretations published by social psychologists emphasized their salience for understanding the behaviors and development of children growing up in a variety of settings (e.g., see One Boy's Day: A specimen record of behavior; Barker and Wright 1951), as well as their impact on the schooling experiences of American youth (e.g., see Big School, Small School: School size and student behavior; Barker and Gump 1964). In each case, the assets and opportunities associated with the nature of the context for students' everyday relational experiences, behavior, and learning were analyzed. Using a variety of case study-like methods, Barker, Wright, and Gump implemented thoughtfully designed studies and published results describing the links among children's contexts and associated experiences.

However, the behavioral science field undertook studies and made reports that involved mainly Midwestern
European American children. Many studies were conducted at the Midwest Psychological Field Station in Oskalooska, Kansas, and other areas around Lawrence, Kansas, given the social scientists' affiliations with the University of Kansas. It is critical to acknowledge that Native Americans were, in fact, present in the settings where these ecological studies were produced but were not included in the published scholarship. In fact, such youth resided in the area and others were enrolled in the nearby Haskell Institute for Native Americans, which began admitting students in 1884. However, the experiences of Native American youth are not identified as having been included in the ecology-linked scholarship produced during that period. Thus, irrespective of opportunity, the penchant to "overlook" or under-plan for diversity is not new, and it has not ended. The current 4-H PYD project was conducted virtually 50 years following the seminal ecological studies by Barker, Wright, and Gump.

As described by Bowers, Geldhof et al., as the opening conceptual frame for their theoretical model, the authors cite Roth and Brooks-Gunn (2003a, b) as a means to introduce the viewpoint that youth development programs aimed at enhancing health and thriving among adolescents concluded that young people should be regarded not as problems to be managed but, instead, as resources to be developed. The Roth and Brooks-Gunn insights built on Larson's (2000) vision for research aimed at understanding and promoting PYD. However, there continues to be a tradition of studies focused on deficit outcomes when including minorities of color; accordingly, the current project represents an unusual focus (PYD) and is impressive in scope. Relative to minorities, as a tradition, research designs continue to ignore social structure-determined assets and maintain a tradition which implies white populations as the healthy standard for informing processes of development. Importantly and counter to this trend, the current collaborations involved in the 4-H Study provide a more equitable goal of exploring PYD, more generally, for diverse adolescents. However, the well-intended effort of this large scale study represents but an intention. The impressive undertaking has produced findings and conclusions about PYD which address the experiences of Euro-American adolescents. The highly problematic aspect of the policy and practice implications is that the conceptual strategies framed by Figure 1 (see Bowers et al., in this volume) leaves many knowledge gaps when it comes to the programmatic strategies actually needed for obtaining PYD for all citizens, including youth of color.

There are also other methodological and conceptual themes that are important to note. As already suggested, another theme is the problem of data sufficiency for analyzing and obtaining discrete, within-group, ethnic-group findings. In turn, still another theme of relevance for all 
PYD articles stems directly from the model provided as Figure 1, and will be addressed as conceptual challenges given the sample size problems previously noted.

Addressing the theme of diversity, the eight waves of the ambitious 4-H PYD sampling strategy represent a significant effort when the sheer number of adolescents sampled is considered (!). Beginning with the first wave in 2002, which included 1700 fifth grade youth and about 1,100 parents from 13 states in the United States, and given subsequent strategies to maintain statistical power, the final sample is impressive. Specifically, and as described in each report when delineating its sampling procedures, given the questions posed and constructs identified according to wave, by the completion of Grade 12 data collection, Bowers, Geldhof et al. noted in their introduction that the 4-H Study involved 42 states and more than 7,000 adolescents and 3,500 parents. Given the large sample size, available constructs, and nuanced variables, the overall study and its related articles represented an opportunity to examine thriving across a decade. However, as acknowledged in the introduction and individually by article authors, overall the sample was homogenous, and the sample size precluded obtaining insights for non-European American adolescents. However, given the expense of the undertaking, sophistication of its design, the resources for project implementation that were expended, and the repeated stating of sampling shortcomings in this research effort, we need to ask, Is acknowledging the problem enough? Importantly, this study made the social science correction for gender inclusion (i.e., given the significant representation of females). It is a valued design implementation. Nonetheless, it will be important for the authors to ponder the steps necessary for obtaining equal and "seamless success" for ethnic diversity inclusivity, as well.

The additional efforts and conditions required for successful follow-up data collection with minority samples is neither an inconsequential concern nor an unexpected dilemma, particularly for research efforts requiring repeated measurements. Myriad relevant research challenges have been addressed both during the latter decade of the twentieth century (e.g., see Graham 1992) as well as in the initial decade of the twenty-first century (e.g., see Fisher et al. 2002). Accordingly, a variety of critiques and suggestions have been offered, and these works provide insights about research tactics and strategies that appear, unfortunately, under-addressed or ignored; accordingly, methodological and conceptual shortcomings persist, independent of whether the funding emanates from private or federal funding sources. The sampling sufficiency concern is the most obvious methodological dispute we have with the 4-H Study. Other conceptual limitations suggested by the model itself (see Figure 1 in Bowers, Geldhof, et al.) introduce additional analysis challenges that add to the sampling difficulties previously described.

As illustrated in Figure 1, defining strengths of adolescents without also considering diverse ways in which social structures and contexts affect youth may under-estimate other markers of strength equally important for PYD characteristics. For example, for youth from economically marginal, ethnically devalued, or undocumented families, demonstrating creativity in coping or thinking about one's attributes given multiple and complex challenges may be critically important for influencing PYD. Thus, missing from the model is the role of proactive and defensive strategies or adaptations (i.e., suggesting the positive attributes of challenge) for practicing and developing adaptive behaviors which offset the impact of risk (i.e., for depression, delinquency, and substance use). Although an uncomfortable acknowledgement for some, as previously noted, America is not yet post-racial. Social structures are designed and function to facilitate chronic conditions of inequality and high-risk conditions which persist both intentionally and unintentionally. Particularly for the life situations of youth experiencing long-term statuses of significant low income, race/ethnicity associated inequality, immigrant status challenges, sibling (or other adult) care requirements, navigation of neighborhoods with limited resources, and expectations for household economy contributions (i.e., all unacknowledged social structural linked situations), these issues matter profusely. Accordingly, understanding genuine supports and resources that ameliorate the impact of excessive challenges are critical steps for promoting PYD equally for all youth. Thus, nuanced understandings are critical for decision makers for ascertaining which supports and assets are actually experienced as authentic, supportive ones.

Significantly coping with challenge may be a norm which may compromise the contributions of ecological assets or, alternatively, serve as a foundation for developing positive youth outcomes by fostering adaptive responses in creative and positive ways. Thus, an opportunity for the model to accommodate and acknowledge successful and unsuccessful behavioral experiences and challenges might serve to assist in identifying additional assets particularly important for youth in exceptional circumstances. For example, currently, the noted contributions and ecological assets that are included omit the role of challenge as an asset. Also important to capture are not only the range of assets, but how such resources are used and whether they offset experienced challenges leading to positive or negative outcomes. In other words, assets vary depending on the individual challenge and their perception or meaning-making and actual experience as such.

Finally, by defining thriving narrowly as positive youth development (five Cs), does it suggest other forms of 
thriving as suboptimal? For example, a teenager's coping with life in a homeless shelter may be thriving but is it the PYD intended and presented in Figure 1? Might the model be informed by considering levels of complexity that more accurately represent lives made intricate by underacknowledged circumstances (e.g., due to under-resourcing or an over-abundance of supports)?

Conceptual clarity and methodological breadth are critically important to ensure that the myriad experiences of diverse youth can be captured as well. An analysis of the articles represented in this special issue suggests that we are on the right trajectory and have come a long way when compared with findings reported fifty plus years ago. As acknowledged by the authors, although it was not possible to provide analyses of the sample's racial cohorts across waves of data collection, nevertheless, and importantly, the findings of the 4-H Study still demonstrate the salience and relevance of a decade long, asset-focused longitudinal study of PYD. The magnitude of the study situates this endeavor in an incomparable and historical place.

In every walk of life in early twenty-first century America, we are neither post-racial nor in a "powerunmotivated" space; in fact, issues of race, ethnicity and diversity are much more nuanced and complex than during previous periods. However, the articles of this special issue indicate that we can apply models of human development to those youth who are racially diverse and thus assess strategies for navigating the distance required for arriving at our goal of PYD for all young people. As suggested by the poem by Robert Frost which follows, although we have made progress, there is still a distance to travel before arriving at the goal of universal PYD.

\author{
"Stopping By Woods on a Snowy Evening," by \\ Robert Frost \\ The woods are lovely, dark and deep, \\ But I have promises to keep, \\ And miles to go before I sleep, \\ And miles to go before I sleep
}

Author Contributions M.B.S. and T.R.S. were invited and participated equally in the review of the articles, conceptualization of the statement, and writing of the submitted Commentary. Both authors read and approved the final manuscript.

\section{References}

Barker, R. G., \& Gump, P. V. (1964). Big school, small school: High school size and student behavior. Stanford, CA: Stanford University Press.

Barker, R. G., \& Wright, H. F. (1951). One boy's day: A specimen record of behavior. New York, NY: Harper.

Fisher, C. B., Hoagwood, K., Boyce, C., Duster, T., Frank, D. A., Grisso, T., et al. (2002). Research ethics for mental health science involving ethnic minority children youth. American Psychologist, 57(12), 1024-1040.

Graham, S. (1992). Most of the subjects were White and middle class: Trends in published research on African Americans in selected APA journals, 1970-1989. American Psychologist, 47(5), 629-639. doi:10.1037/0003-066X.47.5.629.

Havighurst, R. (1953). Human development and education. New York: McKay.

Larson, R. W. (2000). Toward a psychology of positive youth development. American Psychologist, 55(1), 170-183.

McGee, E., \& Spencer, M. B. (2012). Theoretical analysis of resilience and identity: An African American engineer's life story. In E. J. Dixon-Román \& E. W. Gordon (Eds.), Thinking comprehensively about education: Spaces of educative possibility and their implications for public policy (pp. 161-178). New York, NY: Routledge.

Roth, J. L., \& Brooks-Gunn, J. (2003a). What is a youth development program? Identification and defining principles. In F. Jacobs, D. Wertlieb \& R. M. Lerner (Eds.), Enhancing the life chances of youth and families: Public service systems and public policy perspectives: Vol. 2 handbook of applied developmental science: Promoting positive child, adolescent, and family development through research, policies, and programs (pp. 197-223). Thousand Oaks, CA: Sage.

Roth, J. L., \& Brooks-Gunn, J. (2003b). What exactly is a youth development program? Answers from research and practice. Applied Developmental Science, 7, 94-111.

Spencer, M. B. (2011). American identity: Impact of youths' differential experiences in society on their attachment to American ideals. Applied Developmental Science, 15(2), 61-69.

Spencer, M. B., Brookins, G. K., \& Allen, W. R. (Eds.). (1985). Beginnings: Social and affective development of Black children. Hillsdale, NJ: Erlbaum.

Spencer, M. B., \& McLoyd, V. (1990). Special issue (minority child development). Child Development, 61(2), Entire Issue.

Margaret Beale Spencer earned the Ph.D. at the University of Chicago in child and developmental psychology, Committee on Human Development. She is the Marshall Field IV Professor of Urban Education in the Life Course Human Development Program, Department of Comparative Human Development, the College and Committee on Education at the University of Chicago. Spencer's basic research, theory development [Phenomenological Variant of Ecological Systems Theory (P-VEST)], evaluation efforts and collaborative applications posit that all humans are vulnerable (i.e., possess both risks and assets). Scholarship focuses on resiliency and emphasizes strengths, productive coping, positive identity processes and healthy outcomes for culturally diverse youth given myriad challenges. Spencer has authored over 125 articles and chapters, coedited four volumes, is the recipient of funding from over three dozen private and federal sources, and has received broad recognition: Elected membership into the National Academy of Education; SRCD Distinguished Contribution Award; APA Senior Career Award for Distinguished Contributions to Psychology in the Public Interest; Inaugural AERA Fellow; and Fletcher Fellowship recipient for scholarship furthering the goals of the U.S. Supreme Court's Brown v. Board of Education Decision of 1954.

Tirzah R. Spencer is a Senior Research and Evaluation Scientist at Nemours Health and Prevention Services/A.I. duPont Hospital for Children. She received her B.A. in Neuroscience and African American Studies from Oberlin College, her M.P.H. in Social \& Behavioral Sciences and Epidemiology from Boston University, School of Public Health, and her Ph.D. in Biobehavioral Health from the Pennsylvania State University. Dr. Spencer conducts translational 
research emphasizing cultural, contextual, and developmentally specific approaches to assessing trajectories of risk and resilience in health outcomes among youth. She uses both quantitative and qualitative data analysis strategies to assess the development of chronic health conditions by focusing on perceptions of youth and methods of coping in family and community contexts. Areas of focus include the implementation of intervention and evaluation studies. Tirzah Spencer has given lectures and published in the areas of childhood obesity, identity development, and health disparities, particularly among youth and families living in low-income urban environments. 